\title{
Ergonomic Chair Design by Fusing Qualitative and Quantitative Criteria Using Interactive Genetic Algorithms
}

\author{
Alexandra Melike Brintrup, Jeremy Ramsden, Hideyuki Takagi, Member, IEEE, and Ashutosh Tiwari, Member, IEEE
}

\begin{abstract}
This paper emphasizes the necessity of formally bringing qualitative and quantitative criteria of ergonomic design together, and provides a novel complementary design framework with this aim. Within this framework, different design criteria are viewed as optimization objectives, and design solutions are iteratively improved through the cooperative efforts of computer and user. The framework is rooted in multiobjective optimization, genetic algorithms, and interactive user evaluation. Three different algorithms based on the framework are developed, and tested with an ergonomic chair design problem. The parallel and multiobjective approaches show promising results in fitness convergence, design diversity, and user satisfaction metrics.
\end{abstract}

Index Terms-Design optimization, ergonomics, interactive evolutionary computation, multiobjective optimization.

\section{RESEARCH PROBLEM}

W HERE quantitative engineering and qualitative design concepts are used together to deliver a product, they need to be accurately represented, thereby: 1) facilitating taking advantage of human approximate reasoning and qualitative judgment; 2) enabling delivery in cases where an accurate mathematical representation of a design solution search space is not readily available; 3 ) achieving built-in flexibility where definitions of design criteria or their relative importance change; and 4) achieving better problem understanding, which in turn can promote innovative solution discovery.

Typical examples where the two types of views are merged include automotive design, appliance design, and architectural design [1]. However, there exist very few, if any, models that successfully incorporate qualitative and quantitative influences in a formal manner [2]. In ergonomic product design, this gap manifests itself in two ways.

1) A splitting of aims, where ergonomists evaluate designs based on either only qualitative concerns or only quantitative concerns (or a nonformal mixture of both). For example, an account of comfort taken firsthand from the target user might be considered as the only measure of whether a chair is ergonomic. According to another school

Manuscript received June 22, 2006; revised November 14, 2006 and April 23, 2007.

A. M. Brintrup is with the Department of Engineering, University of Cambridge, Cambridge CB2 1RX, U.K. (e-mail: ab702@cam.ac.uk).

J. Ramsden is with the School of Applied Sciences, Cranfield University, Bedfordshire MK43 0AL, U.K. (e-mail: j.ramsden@cranfield.ac.uk).

H. Takagi is with Faculty of Design, Kyushu University, Fukuoka 815-8540, Japan (e-mail: takagi@design.kyushu-u.ac.jp).

A. Tiwari is with the School of Applied Sciences, Cranfield University, Bedfordshire MK43 0AL, U.K. (e-mail: a.tiwari@cranfield.ac.uk).

Digital Object Identifier 10.1109/TEVC.2007.904343 of thought, comfort may not necessarily represent a thorough anthropological analysis, in which the fit of the chair to the posture and size of its user is carried out [3]; this school argues that comfort evaluation might be unreliable and even dangerous at times, since the comfortable but anthropologically unfit chair can lead to medical problems.

2) In a product development cycle where ergonomic design is but one concern among many, lack of formalization of the weighting and interaction of the different criteria needlessly prolong development.

One can exemplify the time lost over negotiating design criteria with a case identified in a survey carried out by the authors and to be published in due course.

When a furniture company sets out to design a chair, the design criteria include but are not limited to maximum comfort scores from test users for a maximum amount of time, maximum resistance to loading, a shape that led to the greatest stack of products in the smallest space possible, and minimum cost of production. The constraints on the design include: size constraints that vary among different population segments for which the design is intended; and some shape constraints that reflect the image of the firm in the marketplace. While some of these criteria and constraints are of qualitative concern, the rest are quantitative.

The responsibility of satisfying the different criteria typically remains within different departments. As formal weightings are not given to different criteria, and the interaction between criteria is left unexplored, the process of design improvement takes place via a lengthy negotiation process between departments. The chair design example clearly demonstrates the way in which qualitative and quantitative criteria are optimized without a formal optimization framework.

This paper is concerned with the development of a novel algorithmic framework to bring qualitative and quantitative design criteria together, in order to facilitate solution search and evaluation. The framework is based on principles of multicriteria decision making and user-interactive evolutionary optimization techniques. Part of the chair design problem is used for testing the framework.

Section II outlines the principles that lay the foundation of the framework. Section III describes the chair design problem with which the algorithms are tested. Section IV describes three algorithms that follow the framework. Section V compares the results obtained from these three algorithms using fitness convergence, diversity, and user preference metrics. Section VI provides key observations and speculates on future developments. Section VII provides the conclusion. 


\section{INCLUSION OF QUALITATIVE DESIGN CRITERIA USING EVOLUTIONARY MULTIOBJECTIVE OPTIMIZATION: A NEW APPROACH}

\section{A. Qualitative Criteria in Design}

Qualitative is a term typically used in opposition to the word quantitative, which refers to numerically representable phenomena whose characteristics are objective and context-independent. The term qualitative, on the other hand, refers to phenomena whose characteristics are best expressed by narrations, opinions and beliefs rather than quantities. Qualitative characteristics are subjective and they may vary according to the domain in which they are handled.

We previously distinguished the different types of qualitative phenomena that occur during the design optimization process, such as multidimensionally and unidimensionally qualitative criteria [1]. This paper focuses on multidimensionally qualitative criteria, even the conceptual meaning of which is difficult to codify and quantify. Adjectives such as ergonomic, pretty, easily handlable, and comfortable are examples of multidimensionally qualitative criteria.

There exist two approaches for supporting multidimensionally qualitative views in design: 1) generalizing the qualitative opinion in a way that reflects the majority view or 2) considering individuality. In today's competitive design world, considerable attention is given to satisfy a diverse range of customer demands, and design customization and market segmentation are practised to serve to individual sensitivities. The work reported in this paper adopts the second view for dealing with multidimensional qualitativeness.

In ergonomic design, the formalization of qualitative criteria and their integration with quantitative criteria can lead to advantages such as flexibility in solution definition and search, reducing design improvement time, better problem understanding, and better solution delivery by taking advantage of human qualitative judgment.

The rest of this section focuses on the principles used to make such an integration possible.

\section{B. Multiobjective Optimization}

Multiobjective ${ }^{1}$ design optimization is defined as the problem of finding a vector of decision variables that optimizes a vector function whose elements represent multiple objective functions. The basis of multiobjective optimization comes from the need to achieve compromise decision-making in a problem of many conflicting objectives. In such an environment, the ideal platform would enable us to gather and present a diverse set of solutions, each with its own offering of different levels of objective satisfaction, so that a choice of solution or solutions can easily be reached. In our novel framework, qualitative influences in ergonomic design are viewed as design criteria, which make multiobjective optimization an ideal base from which to formally define the design problem in terms of its objectives, objective preferences, and constraints. Multiobjective optimization alone does not consider qualitative criteria, or subjective influences on

\footnotetext{
${ }^{1}$ We use "objective" here in the sense of "design goal," "qualitative" for a design goal that is subjective, and "quantitative" for a design goal that is objective.
}

quantitative criteria, as it is a method developed essentially to optimize multiple functions that are quantitatively represented.

\section{Multiobjective Optimization With Genetic Algorithms (GAs)}

Recently, the usage of evolutionary computing (EC) techniques in multiobjective optimization has become popular. A multiobjective optimization problem modeled by EC is termed evolutionary multiple-objective optimization (EMO). The suitability of EC and, particularly, genetic algorithms (GAs), a subset of EC, for multiple-objective optimization is described in [4].

\section{Interactive Genetic Algorithms (IGAs)}

Interactive evolutionary computation (IEC) is an EC method that optimizes a target system based on human subjective evaluation, where the human plays the role of the fitness function of conventional EC [5]. IGAs are a type of IEC based on the principles of GA. Parmee defined IGA broadly as system optimization based on human-machine interaction [6]. When IGA is applied to optimization problems such as engineering design, ergonomic design, or architecture, interaction with a human evaluator facilitates the generation of solutions that incorporate human expertise or intuition without having to explicitly codify them into the optimization platform. Interaction between a user and the system can proceed in many ways depending on the task domain. In addition to assigning the qualitative fitness of an individual design, the user may intervene by choosing elite designs for survival, modifying an individual and reinserting it into the population of designs, and freezing parts of the design with the intention of reducing the search space dimensionality. As opposed to conventional artificial intelligence methods that try to model human subjectivity and qualitative decisions, IGA incorporates the human, not a model of the human, into the optimization system. This simplicity and efficiency makes IGA an ideal candidate method for incorporating qualitative influences into design optimization.

It is important to emphasize that optimization with IGA produces subjectively influenced designs. Alone it does not consider quantitative criteria. The interaction of the user is required to drive the GA itself. Since interaction with the user is used as the fitness function, this inevitably brings subjectivity into the optimization process. This is so, even when the user assesses the design from a mathematically representable, i.e., quantitative, objective.

\section{E. A Review of Previous Approaches for Integrating Multidimensional Qualitativeness in Quantitative Optimization}

One method for handling multidimensional qualitative views is to represent them quantitatively according to the individual preferences of the designer, who articulates his/her qualitative design objectives, and, considering how the objective is concretely realized, describes objective preferences and the reasoning behind them. This description is then incorporated into the EMO problem quantitatively using fuzzy modeling. There is a lack of flexibility should the designer change his/her mind or should a new subobjective need to be incorporated, since the fuzzy model then needs to be readjusted. 
The above procedure of using fuzzy modeling in EMO to represent qualitativeness has been proposed by Oduguwa et al. [7]. They suggested that for every quantitative solution lying on the Pareto front, there exists a corresponding qualitative evaluation expressing the designer's opinion about the problem, and that this qualitative evaluation stands in a unique relation with the quantitative solution. The approach mainly deals with quantitative and qualitative information that are inherently conflicting. The authors reported that many qualitative objectives increase fragmentation of search space.

Parmee et al. [6] have developed the interactive evolutionary design system (IEDS), where a rule-based preference component of the algorithm allows the designer to interactively express his/her preferences in terms of natural language, which then direct the MOO search into compromise regions. Software agents monitor the coevolutionary processes, and the satisfaction of objectives and constraints using a designer preference rule base. In both Oduguwa et al. and Parmee et al.'s approaches, multidimensional qualitative data were not handled.

Another previous approach for integrating qualitativeness into optimization is partial interaction. In the nurse scheduling system developed by Inoue et al. [8] the optimization functions are quantitative. A Pareto-based vector-evaluated genetic algorithm evolves a set of solutions. The nurse interacts with the system after the solutions are generated by this quantitative optimization. He/she views and modifies the scheduling solutions and reinserts them into the population.

In a similar system developed for molecular docking, users are able to modify the GA-generated solutions [9]. The GA evolved a quantitative function for a number of generations. Then, users attempt to dock a GA-generated ligand manually in a virtual reality environment. This solution then replaced the worse solution in the population found by the GA. They reported that solutions that did not use interaction were better than solutions with interaction. This was due to the limitations of the virtual reality environment and the large number of atoms, which made it difficult for the user to realize which part of the molecule was being viewed during docking. This system did not use multiobjective optimization, nor did it use user-assigned fitness in the GA.

An interesting method is the collaborative project scheduling system proposed by Shackelford and Corne [10]. Here, the quantitative fitness of a schedule is first calculated. The schedules are then presented to the user in order of that quantitative fitness. To each, the user assigns a qualitative fitness score, which is then used to breed the next generation. In other words, quantitative fitness score is not used to breed the next generation. These workers reported that the evolutionary scheduler outperformed standard quantitative criteria-based industry packages in terms of user satisfaction. However, difficulty in the evaluation of solutions was a concern, which suggests that a more integrative approach to qualitative and quantitative criteria might indeed improve evaluation.

However, another approach to handle multidimensional qualitativeness is Kamalian et al.'s microelectromechanical systems (MEMS) design platform [11]. Here, IGA was used as a single objective optimization method, after the generation of tradeoff solutions with a multiobjective genetic algorithm that optimized designs according to various quantitative objectives. Their system showed that the preferences of designers were significantly oriented towards designs that were optimized by the IEC-enhanced system, compared with the designs optimized without IEC.

We have ourselves previously proposed an alternative framework where the user provides interactive fitness assignments for multidimensionally qualitative design views. The multidimensionally qualitative design views are then treated as qualitative objectives, the attainment of which is optimized along with the quantitative design objectives using an EMO algorithm [1], [12], the well-known nondominated sorting genetic algorithm 2 (NSGA 2). We also developed and tested a parallel IGA and a sequential IGA as alternative EMO approaches for optimizing multidimensionally qualitative and quantitative objectives together [13].

Machwe et al. have also subsequently employed a similar approach to bridge design [14]. In their system, a multidimensional qualitative criterion was integrated as an objective to the EMO. The EMO algorithm used was the strength Pareto evolutionary algorithm (SPEA). Some of the user scoring was given by software agents. A machine learning system was proposed to reduce the burden on the user.

In Machwe et al.'s most recent work, a mutation-only evolutionary program attempted to optimize a weighted sum function based on five criteria, two of which were rule based- or user-given aesthetic criteria, while the rest were quantitatively evaluated structural criteria [15]. They reported that when the user-given criterion was set to the maximum weight, 2 out of 12 figures survived into the final population, which would not have been the case if user-given criteria had not been included. The structural performance of these solutions in the overall search space was not reported. However, it was argued that conceptual design using this approach is acceptable and any structural problems that may arise could be solved with the introduction of appropriate materials or skeletal frameworks at a later stage. They concluded that further development of machine-based aesthetic assessment is essential.

In our work, we affirm that the interactively given user criteria do not hinder other criteria, and result in a compromise decision that satisfies all design constraints with the best possible blend. It is evident that user interaction during multicriteria design optimization results in innovative and good solutions. In order to gain insight into the means of reaching optimum designs that blend quantitative and qualitative criteria, further research on the mechanisms of compromise decision-making is essential.

To establish such a compromise decision-making mechanism, first, it is important to differentiate the types of qualitative criteria and develop different frameworks to target them [1]. Second, new EMO-based approaches capable of accommodating qualitative-quantitative compromise decision-making need to be developed and tested. In this paper, in contrast to all the previous approaches, we apply and develop our novel EMO-based sequential, parallel, and multiobjective IGAs, and apply them to ergonomic chair design. The sequential IGA approach simulates a commonly deployed optimization method for multiple objectives. The multiobjective IGA approach modifies the NSGA 2. The parallel IGA approach is an alter- 
native approach for EMO. The algorithms are collected under the interactive multiobjective design optimization (IMODO) framework explained in the next section.

In this paper, in contrast to all the previous approaches, we apply and develop our sequential, parallel, and multiobjective IGAs to ergonomic chair design. The sequential IGA approach simulates a commonly deployed optimization method for multiple objectives. The multiobjective IGA approach modifies the NSGA 2. The parallel IGA approach is an alternative approach for EMO. The algorithms are collected under the IMODO framework explained in the next section.

\section{F. Interactive Multiobjective Design Optimization (IMODO) Framework}

The IMODO framework is composed of three components.

1) The EMO platform, which optimizes qualitative and quantitative objectives together based on GA principles and follows a multiobjective optimization philosophy.

2) User interaction, which involves the designer acting as the qualitative fitness evaluation function. The designer can dynamically change constraints and preferences between objectives. The designer can modify solutions and reinsert them into the system, and freeze different features of the design at hand.

3) Computer-generated solutions, i.e., the quantitative fitness evaluations performed by the computer through a built-in function.

\section{The Chair Design Problem}

Posture is simply the position of the body during an activity (including resting). An awkward posture is associated with an increased risk of injury. It is considered that the more a joint deviates from the neutral (natural) position, the greater the risk of injury [16].

Ergonomic chair design is sometimes evaluated by measurements on how the chair fits to a given percentage of parts of the body in a given posture (i.e., reclining, working etc.), sometimes by live experiments in which a sitter's feeling of comfort is recorded, or often by a mixture of both. In the present study, we define the ergonomic chair design problem as the problem of finding an optimum set of parameters that control the shape of the chair with respect to a given posture.

Two types of objectives are included in the problem: a qualitative objective based on the designer's evaluation of how suitable the chair looks for a particular posture, and a quantitative objective that measures how closely the chair fits to the sitter's posture.

For the purpose of simplicity, the algorithm starts from random solutions and not pre-encoded chromosomes that represent existing chairs (although would be possible with our framework). Again for simplicity, preferences, i.e., weighting between objectives, are kept equal. The user interaction is only through ratings, and the user does not modify parameter or constraint values during the optimization process

The design variables are shown in Fig. 1 (upper). The headrest, backrest, angle of backrest to seat, footrest, angle of footrest to seat, and seat height are included, while the armrest, seat contour, and cushioning are not taken into account for simplicity. A real valued string is encoded.

In terms of representation, the real-coded chromosome consisted of dimensional chair parameters shown in Fig. 1. Ten control points were used for the B-spline, followed by centripetal spline interpolation.

The following objective functions were defined.

Objective function 1

$$
f_{1}(c, l)=f_{\text {user }}(c, l)
$$

where the user given rating from a scale of $0-9$ denotes the user's comfort $(c)$ and liking $(l)$ of the design.

Objective function 2, shown in (2) at the bottom of the page.

The parameters used in the quantitative objective function are shown on Fig. 1 (lower part), where ch_spine and $p$ spine are $n$ B-spline points denoting a B-spline approximation of the chair's backrest spine and the person's spine.

- ch1 is the chair's headrest midpoint;

- $p 1$ is the person's head midpoint projected to the back of head;

- ch2 is the chair's knee point;

- $p 2$ is the person's knee point;

- ch3 is the chair's end of footrest point;

- $p 3$ is the person's end of footrest point.

The following constraints were coded.

- constraint $[0]=90<$ chair knee angle $<$ person knee angle (in degrees);

- constraint $[1]=90<$ chair headrest angle $<180$;

- constraint [2] $=90<$ chair angle of backrest to seat $<$ 180.

The seated human body's positional data is a predefined and fixed model, which is based on the 99 percentile white male body [17]. As examined above, multiple objectives, multiple

$$
\begin{aligned}
f(x, y)= & \left(\sum_{i=1}^{n} \sqrt{\left(\text { ch_spine }_{\mathrm{ix}}-p_{\text {spine }} \mathrm{ix}\right)^{2}+\left(\text { ch_spine }_{\mathrm{iy}}-p_{\text {spine }} \mathrm{iy}^{2}\right)^{2}}\right) \\
& +\sqrt{\left(\operatorname{ch} 1_{x}-p 1_{x}\right)^{2}+\left(\operatorname{ch} 1_{y}-p 1_{y}\right)^{2}} \\
& +\sqrt{\left(\operatorname{ch} 2_{x}-p 2_{x}\right)^{2}+\left(\operatorname{ch} 2_{y}-p 2_{y}\right)^{2}} \\
& +\sqrt{\left(\operatorname{ch} 3_{x}-p 3_{x}\right)^{2}+\left(\operatorname{ch} 3_{y}-p 3_{y}\right)^{2}}
\end{aligned}
$$




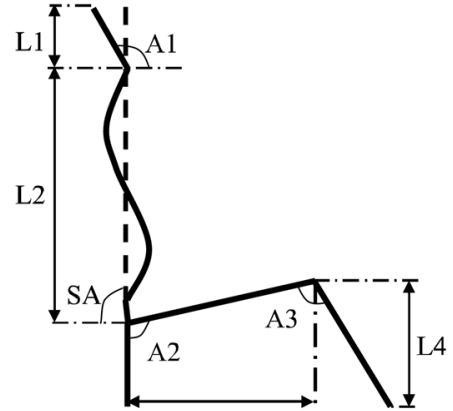

L3

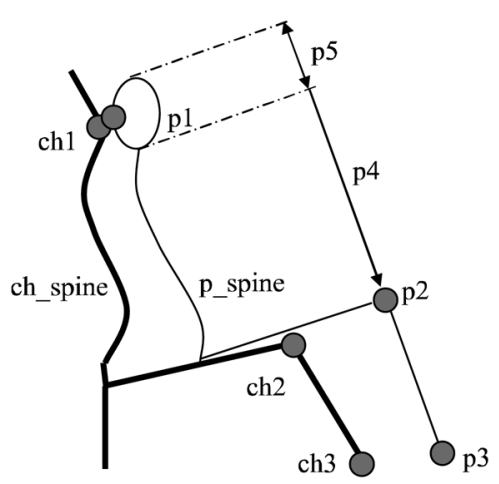

Fig. 1. Upper part illustrates chair design parameters while lower part shows objective function parameters.

constraints, and a mixture of qualitative and quantitative objectives all constitute the features of the chair design problem. The problem, therefore, is ideal for testing the framework proposed in Section II-F. The following section outlines three different algorithmic approaches that make use of the proposed framework. The results are outlined and discussed in Section V.

\section{Multiobjective Chair Design With IGAs}

\section{A. Sequential IGA}

The sequential IGA deals with multiple objectives by taking turns to optimize the quantitative and qualitative objectives independently.

A single population is evolved by a sequentially switched fitness function. An initial qualitative run is performed with a conventional IGA. Users are allowed to give the same rating to more than one design. The qualitatively evaluated run is followed by a given number of quantitative optimization runs, where fitness is evaluated by a regular fitness function.

The individuals created from a qualitative run are fed into the following quantitative run as parent designs, ensuring that the start point for the quantitative run is the qualitatively optimized designs. This is how the connection between qualitative and quantitative criteria is ensured.

With this algorithm, we aimed to represent a typical design cycle in a design firm, where the design is "thrown over the wall" between the marketing department that is concerned with qualitative aspects of the design, and the R\&D Department that is concerned with quantitative aspects. Fig. 2 shows the flow of sequential IGA.

\section{B. Parallel IGA}

EC techniques are suitable for parallelization, since crossover, mutation, and evaluation can be performed independently. It is possible to separate individuals themselves to be evolved in separate locations or programs to enable different selection and recombination routines for each individual. The reason for choosing parallelization depends very much on the problem at hand. Parallelization can be a solution for computationally demanding problems, for applying different selection and recombination routines to individuals, or for separating populations, as in our case. In any of these cases, three main parallelization techniques are widely used [18].

1) Master-slave parallelization, where a single processor maintains control over selection and uses the other processors only for crossover, mutation and evaluation of individuals. This technique is useful for few processors and very large evaluation times.

2) Island model, where every processor runs an independent EC. The processors cooperate by regularly exchanging elite migrants. This technique is suitable for clustering populations.

3) Diffusion model, where the individuals mate with other individuals only in the local neighborhood. This technique is particularly suitable for massively parallel computers with a fast local intercommunication network.

The use of parallelization on EMO can be effective as the goal of EMO is to find a set of good solutions rather than a single optimum, and there is a limited amount of literature on the use of parallelization in EMO [19], [20]. On the other hand, the use of parallelization techniques in IEC has not been reported except in our previous work [13].

We propose that the use of parallelization and interactivity together is advantageous and natural for our problem. Solutions to multiple objectives can be evolved in separate populations with elite migrants exchanged between one another, i.e., following the island model. The qualitative fitness of a solution on the qualitatively evaluated population island $\left(\mathrm{P}_{\mathrm{QL}}\right)$ is obtained through user interaction, while another population on the quantitatively evaluated population island $\left(\mathrm{P}_{\mathrm{QN}}\right)$ is evolved through a regular fitness function. With this method, a compromise decision can be encouraged by the migration of elites between populations. However, it takes much longer for a user to evaluate designs than a computer. If the objectives are optimized on separate populations, the $\mathrm{P}_{\mathrm{QN}}$ can have more iterations, while the $\mathrm{P}_{\mathrm{QL}}$ is optimized. Similarly, a bigger population size can be used with $\mathrm{P}_{\mathrm{QN}}$ resulting in faster convergence, whereas this is generally not possible with $\mathrm{PQL}$ due to the burden on the user.

The features of the parallel IGA are as follows.

1) Parallelization technique: the parallel IGA uses an island model and optimizes $n$ separate populations with $n$ separate objectives with immigrants exchanged among them. In our experiments, we use $n=2$.

2) Immigrant selection: the top three elite solutions are selected from each population and forced to migrate.

3) Replacement strategy: the least performing three individual solutions from each population are replaced with the immigrants. 


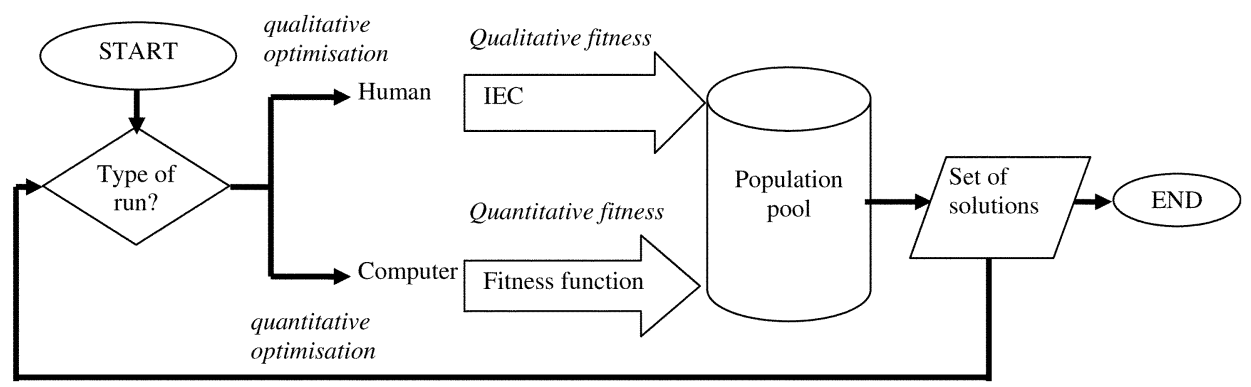

Fig. 2. Sequential IGA procedure.

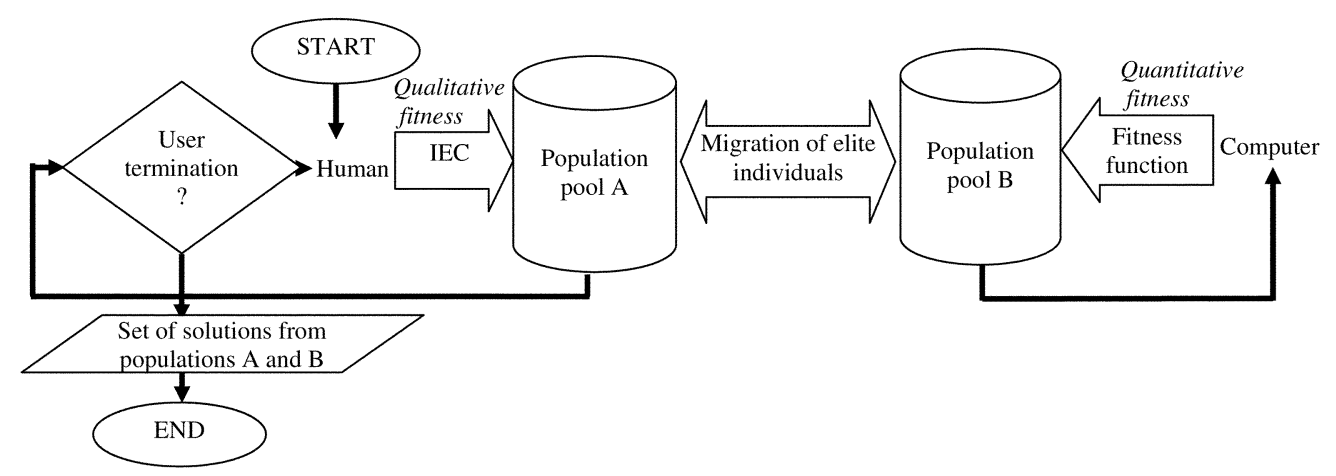

Fig. 3. Parallel IGA procedure.

4) Strategy of fitness assignment to immigrants: In $\mathrm{P}_{\mathrm{QN}}$, immigrants are sorted with respect to their user-given rating. If any two ratings are equal, sorting is done using the calculated quantitative objective fitness. After sorting, an arbitrary quantitative fitness is assigned to the immigrants to ensure their survival. The arbitrary fitness assignment proceeds as follows.

1) Sort immigrants according to qualitative fitness, in descending order, i.e., the individual with the best qualitative fitness ranks first.

2) If any two qualitative fitness values are equal, sort those that are equal according to quantitative fitness.

3) Assign the current generation's best quantitative fitness value to the immigrant with rank 1 .

4) Assign $110 \%$ of the current generation's best quantitative fitness value to the immigrant with rank 2 .

5) Assign $120 \%$ of the current generation's best quantitative fitness value to the immigrant with rank 3 .

In $\mathrm{P}_{\mathrm{QL}}$, immigrants are all given the minimum, i.e., the best, qualitative fitness. The reason is that the qualitative fitness rating is a discrete value and designs taking the same rating are allowed. However, it is very unlikely that any two designs would be assigned the same quantitative fitness after (computer) evaluation. On the other hand, even though two designs may differ from each other, the user might give them the same rating. Fig. 3 shows the flow of the parallel IGA procedure.

\section{Multiobjective IGA}

Multiobjective IGA is based on a modified version of a popular multiobjective optimization algorithm, the nondominated sorting GA 2 devised by Deb et al. [21]. This algorithm enhances the usual nondomination-based multiobjective optimiza- tion techniques by introducing the concepts of elitism and diversity. Elitism ensures the preservation of globally good solutions from generation to generation. Diversity ensures achieving a set of well-spread solutions in the objective space.

In the nondominated sorting GA 2, elitism is achieved by combining parent and offspring populations before sorting them for nondomination. Nondomination of a solution shows how many solutions are better in all criteria then the current solution. The nondominated sorting GA 2 also tries to obtain diverse solutions on the Pareto front by performing the so-called crowding distance calculation. Reference [21] contains a detailed description of the nondominated sorting GA 2 procedure.

We included interactive fitness assignments in the nondominated sorting GA 2, and renamed the algorithm multiobjective IGA. The qualitative objective rating for a solution is obtained from the user, whereas the quantitative fitness of a solution is assessed by the built-in fitness function. Fig. 4 shows the flow of multiobjective IGA.

\section{EXPERIMENTAL RESULTS AND COMPARISON}

\section{A. Experimental Parameters}

We carried out six runs for each algorithmic approach. All algorithms were real-coded, and used tournament selection, mutation with a rate of 0.01 and a distribution index of 10 , and one-point simulated binary crossover with a rate of 0.9 and a distribution index of 20 . The distribution index is any nonnegative real number, which is used to derive the probability distribution in turn used to create a child solution. A large distribution index results in a higher probability for creating near-parent solutions and, conversely, a small value allows distant solutions to be selected. These parameters were kept constant throughout to 


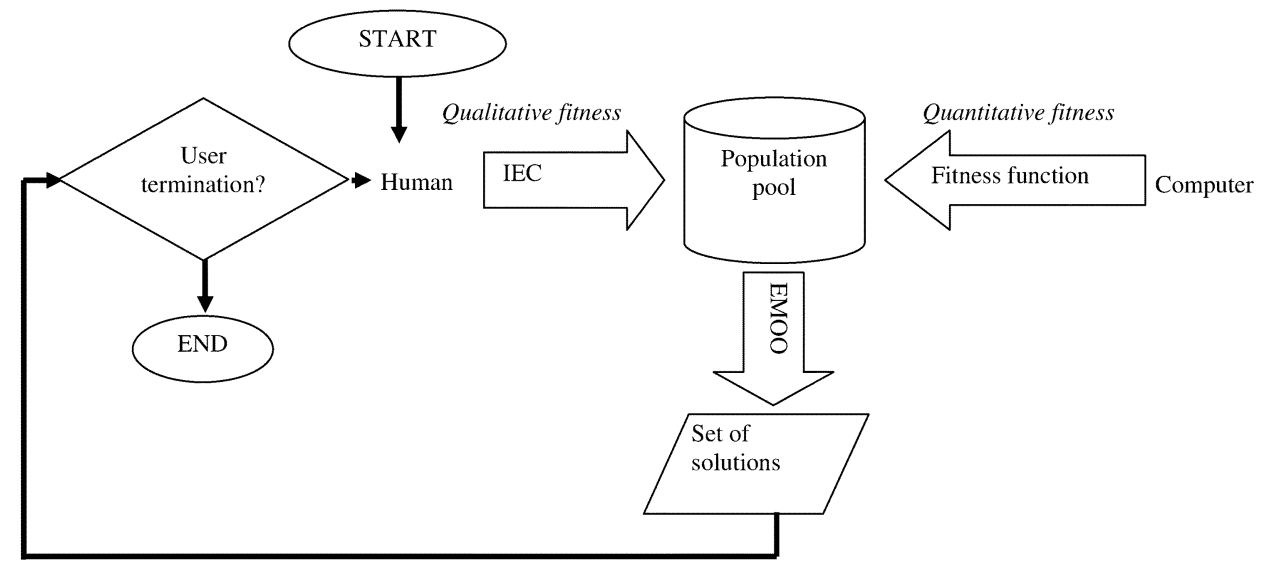

Fig. 4. Multiobjective IGA.

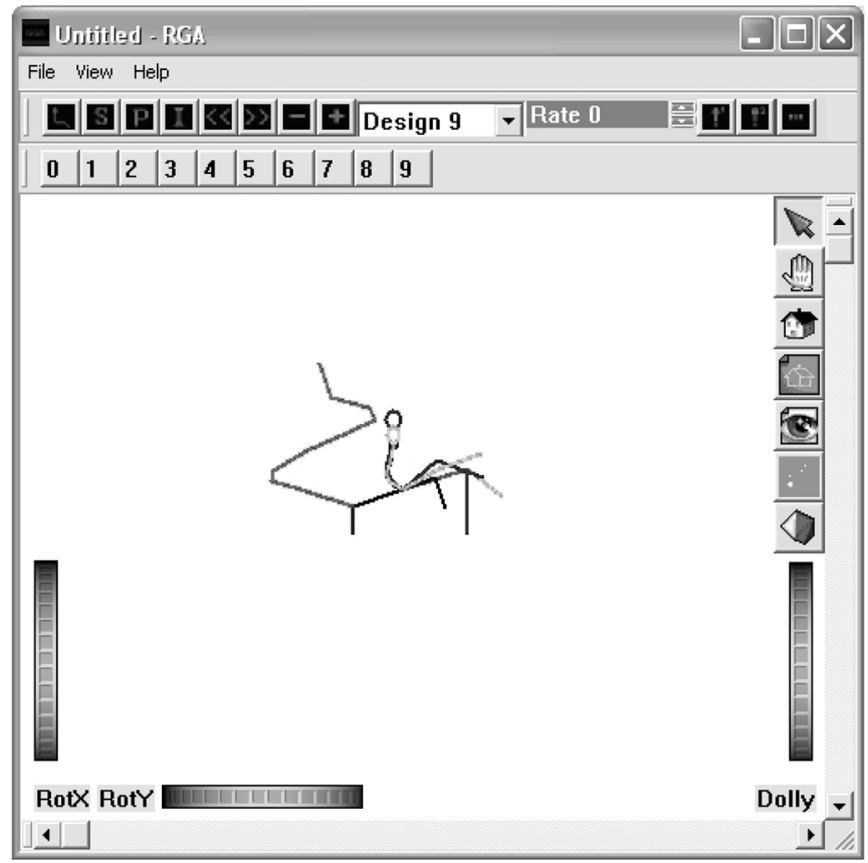

Fig. 5. Graphical user interface of the sequential IGA chair design platform. The interface includes various viewing functions such as zooming, panning, light adjustment, and other adjustment functions such as anthropological data entering, and B-spline parameter editing. The user enters qualitative fitness using the scoring toolbar. The GA parameters can also be adjusted via the interface.

allow a valid comparison of the three approaches. Each of the six runs of any given algorithm started with a different randomization seed, which was reused in the other algorithms.

Human users were two females and three males of ages 24-32, whose expertise ranged over product design, design engineering, and aerospace engineering. The users continued to run the programs until qualitative generation 6 was reached. Each user conducted one test for sequential IGA, parallel IGA, and multiobjective IGA. The graphical user interface is shown in Fig. 5.

The number of generations and the population sizes for each algorithm is given in Table I.
The rest of this section describes the performances of the sequential IGA, parallel IGA, and multiobjective IGA, under three performance metrics, namely, fitness convergence, diversity, and user preference.

Fitness convergence is chosen as it is the most common metric used to evaluate the optimization capabilities of genetic algorithms. However, this metric is mainly used in quantitative criteria problems. As the research problem described in this paper involves qualitative criteria, we included two additional metrics to provide a more accurate comparison between the results. The diversity metric comes from the multiobjective optimization philosophy. It is ideal to obtain solutions that are optimal with a blend of criterion satisfaction, as well as being diverse, so that the decision-maker is offered as much a variety of choices as possible. The user preference metric comes from the need to assess the ultimate success of qualitative concerns in the very domain from which it has originated - that of the user.

\section{B. Fitness Convergence}

Sequential IGA, parallel IGA, and multiobjective IGA were compared over 55, 5, and 55 generations, respectively, in terms of: 1) overall average qualitative fitness; 2) overall average quantitative fitness; 3 ) average qualitative fitness of the last generation; and 4) average quantitative fitness of the last generation. Fig. 6 shows sample individuals obtained at generations 1, 3, and 5 from the multiobjective IGA approach. Table II shows the results of the Wilcoxon signed rank test, an experimental procedure commonly applied to assess user response when evaluating interactively evolved systems. A previous application includes [22], where physiological data response to changes in environment was used as a fitness measure to evolve artificial environments. This nonparametric pair observation test is used to compare average and final quantitative and qualitative fitness values obtained from each algorithm with the aim of concluding which algorithm results in significant success. In our work, for instance, multiobjective IGA was significantly more successful in obtaining the best average qualitative fitness compared with parallel IGA. A risk factor was used to assess the significance of conclusions: a 
TABLE I

Population Sizes, Numbers of Generations, and User Evaluations Per Algorithm

\begin{tabular}{lcccc}
\hline \hline & $\begin{array}{c}\text { Population } \\
\text { size }\end{array}$ & $\begin{array}{c}\text { Number of generations } \\
\text { at each run }\end{array}$ & $\begin{array}{c}\text { Total number of } \\
\text { generations }\end{array}$ & $\begin{array}{c}\text { Total number of } \\
\text { user evaluations }\end{array}$ \\
$\begin{array}{l}\text { Sequential IGA - Quantitative } \\
\text { optimisation run }\end{array}$ & 12 & 10 & 50 & 0 \\
$\begin{array}{l}\text { Sequential IGA - Qualitative } \\
\text { optimisation run }\end{array}$ & 12 & 1 & 5 & 60 \\
$\begin{array}{l}\text { Parallel IGA - Quantitatively } \\
\text { optimized population island }\end{array}$ & 50 & 10 & 50 & 0 \\
$\begin{array}{l}\text { Parallel IGA -Qualitatively } \\
\text { optimized population island }\end{array}$ & 12 & 1 & 5 & 60 \\
Multi-objective IGA & 12 & 1 & 5 & 60 \\
\hline \hline
\end{tabular}

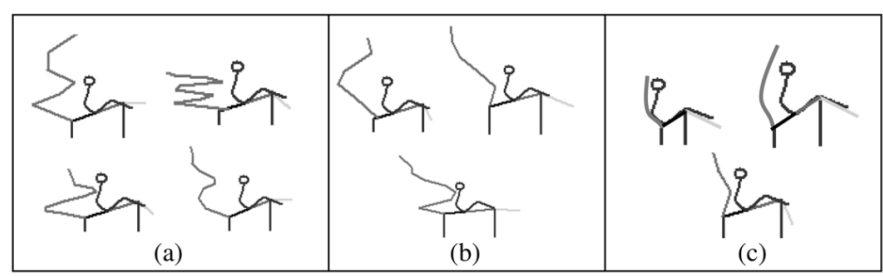

Fig. 6. Sample individuals obtained from multiobjective IGA at (a) generation 1 , (b) generation 3, and (c) generation 5. Different colors are used to highlight different parts of the chair.

factor of $5 \%$ means that the comparative round was in favor of the winner with a significance of $95 \%$.

Parallel IGA obtained the best quantitative convergence and a better qualitative trend than the sequential IGA. The peaks in the quantitative objective convergence illustrate the generations when qualitatively superior immigrants were received, which indicates a conflict between the qualitative and quantitative objectives.

Fig. 7 shows the average fitness values obtained at each generation of each algorithm.

In the sequential IGA, the average qualitative fitness showed a worsening trend over the five runs, while the quantitative results showed a smoothly improving trend.

The best qualitative convergence, and good convergence in quantitative criteria, were obtained with the multiobjective IGA. The fitness improved with respect to both objectives and convergence to the Pareto front was observed.

The multiobjective IGA attained designs that were satisfactory to the user in five generations, whereas the sequential IGA failed to reach an equally satisfactory design in a total of 50 generations. The parallel IGA showed some satisfactory designs within five generations in $\mathrm{P}_{\mathrm{QL}}$.

Although the final quantitative fitness scores were better in the sequential IGA and the parallel IGA than the multiobjective IGA, the sequential IGA performed significantly worse than other two IGAs when the qualitative objective is considered. Rather than reaching a compromise decision between qualitative and quantitative factors, the sequential IGA set the two objectives in competition against each other; resetting and trying to recover from the effects of the opposing objective each time.

A point to bear in mind is the number of generations pursued with each algorithm. The multiobjective IGA pursues one quantitative and one qualitative generation together at a time, since the evolution is simultaneous. As the user must be involved in every generation, only five quantitative generations could be evolved due to user fatigue. Even so, the finally attained quantitative objective was better than that achieved by the sequential IGA at its fifth generation. This is attributed to the criteria being optimized together for five generations as opposed to the sequential optimization philosophy followed in sequential IGA. During the fifth quantitative run, the sequential IGA still tries to rid itself of the effects of the qualitative run it performed earlier.

The superior quantitative convergence of the parallel IGA is attributed to its bigger population size (50 individuals). Such a large population could not be introduced in the other two algorithms as only one population is evolved as opposed to two in the parallel IGA.

A final point to consider is the difficulty of obtaining a smooth convergence in the qualitative criteria due to human inconsistency and intergenerational scoring. When using IGA, a mathematically consistent rating that impacts in proportion or in accordance with changes in the qualitative space cannot be guaranteed. Possible reasons for this might be simply the user not remembering older designs for an accurate comparison, or not being able to provide a rating that can accurately represent this comparison with a score. Intergenerational scoring occurs when the user compares the designs only within the current generation. Intragenerational rating occurs when the user evaluates the designs with respect to all her designs seen so far; the latter places a heavy burden on the user and can lead to inconsistent scoring. In this case, a slight improvement in the overall fitness can still be observed, but the user preference metric tells us more than qualitative criteria convergence.

\section{Diversity}

After the third quantitative run (i.e., the 30th quantitative, 33rd overall generation) of the sequential IGA, the design reached a quantitative optimum, all available designs having then little or no difference (users reported difficulty in distinguishing the designs). Even though minor differences still existed, they were difficult to visualize and evaluate comparatively. This led the users to give similar ratings to all the designs, and it became difficult for the algorithm to diversify them. On the other hand, the diversity-preservation mechanism in the multiobjective IGA provided results that were visually distinct from each other. Although no quantification of diversity 
TABLE II

Comparisons of the Parallel, Sequential and Multiobjective IGAs in Terms of THEIR Quantitative AND QuaLITATIVE Fitness CONVERGENCES

\begin{tabular}{lccc}
\hline \hline & $\begin{array}{c}\text { Sequential IGA vs. } \\
\text { Parallel IGA }\end{array}$ & $\begin{array}{c}\text { Sequential IGA vs. } \\
\text { Multi-objective } \\
\text { IGA }\end{array}$ & $\begin{array}{c}\text { Multi-objective IGA } \\
\text { vs. Parallel IGA }\end{array}$ \\
$\begin{array}{l}\text { Average qualitative fitness } \\
\text { Average quantitative }\end{array}$ & $\begin{array}{c}\text { Sequential IGA with a } \\
\text { risk of 7\% } \\
\text { fitness }\end{array}$ & $\begin{array}{c}\text { Multi-objective IGA } \\
\text { with a risk of 5\% }\end{array}$ & $\begin{array}{c}\text { Multi-objective IGA } \\
\text { with a risk of 5\% }\end{array}$ \\
Final quantitative fitness IGA with a risk & $\begin{array}{c}\text { Sequential IGA with } \\
\text { of 5\% }\end{array}$ & $\begin{array}{c}\text { Parallel IGA with a } \\
\text { risk of 5\% }\end{array}$ \\
Final qualitative fitness & $\begin{array}{c}\text { Parallel IGA with a risk } \\
\text { of 5\% }\end{array}$ & $\begin{array}{c}\text { Sequential IGA with } \\
\text { a risk of 5\% }\end{array}$ & $\begin{array}{c}\text { Parallel IGA with a } \\
\text { risk of 5\% }\end{array}$ \\
& $\begin{array}{c}\text { Sequential IGA with a } \\
\text { risk of 12\% }\end{array}$ & $\begin{array}{c}\text { Multi-objective IGA } \\
\text { with a risk of 5\% }\end{array}$ & $\begin{array}{c}\text { Multi-objective IGA } \\
\text { with a risk of 5\% }\end{array}$ \\
\hline \hline
\end{tabular}

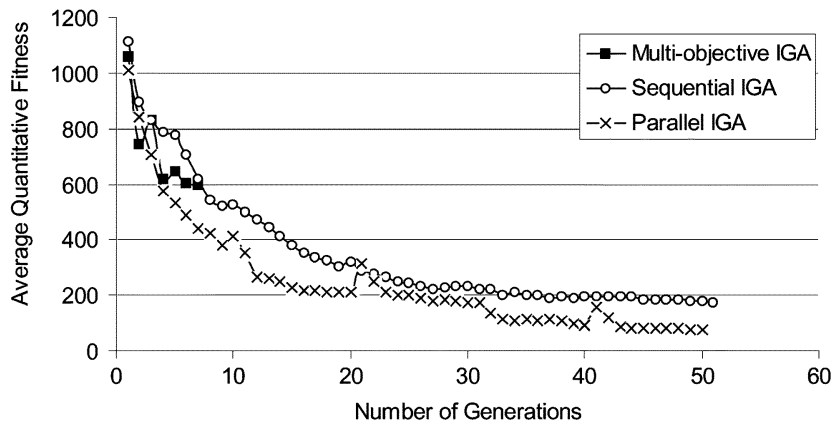

(a)

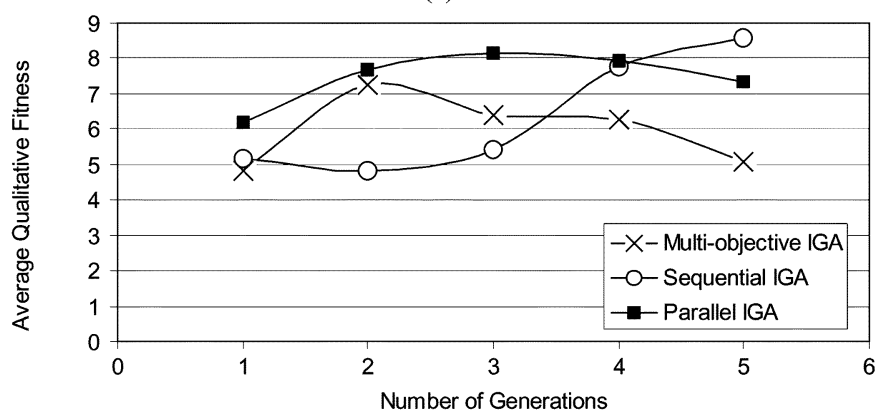

(b)

Fig. 7. Change of average (a) quantitative and (b) qualitative fitness in sequential IGA, multiobjective IGA, and parallel IGA.

was performed, increasing diversity was evidently obtained from sequential IGA, parallel IGA, to multiobjective IGA.

\section{User Preference}

Table III shows the preferences of users on the final set of design solutions obtained from each algorithm. $\mathrm{A}(+)$ sign indicates that a particular user mostly preferred the designs obtained by one algorithm over the other algorithms with which it was being compared. The preference scores were obtained by pairwise comparison. Overall, multiobjective IGA solutions yielded the most preferred results, followed by parallel IGA and then sequential IGA.

In contrast, Machwe reported that it was difficult to steer the path of evolution to the qualitative fitness in his interactive EMO application to bridge design [23]. Our results show that although the parallel IGA provided the user with a bigger selection of final generation designs to choose from, the multiobjective IGA gave better user steering towards the qualitative fitness than the sequential and the parallel IGA. This could be due to the taskdependant conditions used in the multiobjective algorithm.

\section{COMPARISON With User Given FitNess FunCtion}

In addition to experimentation with direct user fitness assignment, we also pursued experimentation with a user-given evaluation function. The goals of this experimentation are to analyze how inconsistency affects the qualitative fitness convergence, and to comparatively analyze the behavior of the algorithms.

We asked the users to describe their qualitative criteria for evaluating the chair. The following criteria were gathered from the users:

1) symmetrical chair legs;

2) minimum difference between chair back length and user's back length;

3) minimum difference between chair front leg length and user's front leg length;

4) minimum difference between chair head rest length and user's head length.

With the response we formulated the following objective function:

$$
\begin{aligned}
f(x, y)= & \left(\sum_{i=1}^{n} \sqrt{\left(c h 2_{\mathrm{ix}}-p 2_{\mathrm{ix}}\right)^{2}+\left(\operatorname{ch} 2_{\mathrm{iy}}-p 2_{\mathrm{iy}}\right)^{2}}\right) \\
& +|A 2-A 3|+|L 2-p 4|+|L 1-p 5| .
\end{aligned}
$$

This objective function replaced the objective function 1 outlined in Section III.

The results in Fig. 8 show the average fitness values obtained at each generation of each run.

It is apparent that without user inconsistency or the effect of intra/intergenerational scoring, qualitative fitness displays a smoother convergence than if fitness is interactively assigned by the user. The persistence of the qualitative objective worsened the quantitative fitness convergence of the parallel IGA. The peaks in the quantitative fitness convergence appear to be more dramatic than when interactive user evaluation is used. The qualitative objective is more consistent without interactive user evaluation, which could explain the higher peaks. The multiobjective IGA displays the worst qualitative and quantitative fitness convergence, which is expected as this algorithm takes more time to optimize both objectives together. When the 
TABLE III

USER PREFERENCES OF FINAL DESIGN SOLUTIONS

\begin{tabular}{lccccc}
\hline & User 1 & User 2 & User 3 & User 4 & User 5 \\
$\begin{array}{l}\text { Parallel IGA (+) vs } \\
\text { Sequential IGA ( - ) }\end{array}$ & $(+)$ & $(-)$ & $(-)$ & $(+)$ & $(+)$ \\
$\begin{array}{l}\text { Multi-objective IGA (+) vs } \\
\text { Parallel IGA ( - ) }\end{array}$ & $(+)$ & $(+)$ & $(+)$ & $(-)$ & $(+)$ \\
$\begin{array}{l}\text { Multi-objective IGA(+) } \\
\text { vs Sequential IGA ( - ) }\end{array}$ & $(+)$ & $(+)$ & $(+)$ & $(+)$ & $(+)$ \\
$\begin{array}{l}\text { Overall } \\
\text { Multi-objective } \\
\text { IGA }\end{array}$ & $\begin{array}{c}\text { Multi-objective } \\
\text { IGA }\end{array}$ & $\begin{array}{c}\text { Multi-objective } \\
\text { IGA }\end{array}$ & $\begin{array}{c}\text { Parallel } \\
\text { IGA }\end{array}$ & $\begin{array}{c}\text { Multi-objective } \\
\text { IGA }\end{array}$ \\
\hline \hline
\end{tabular}

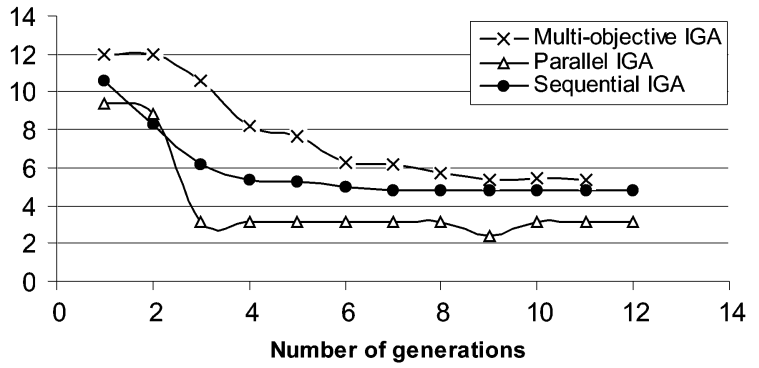

(a)

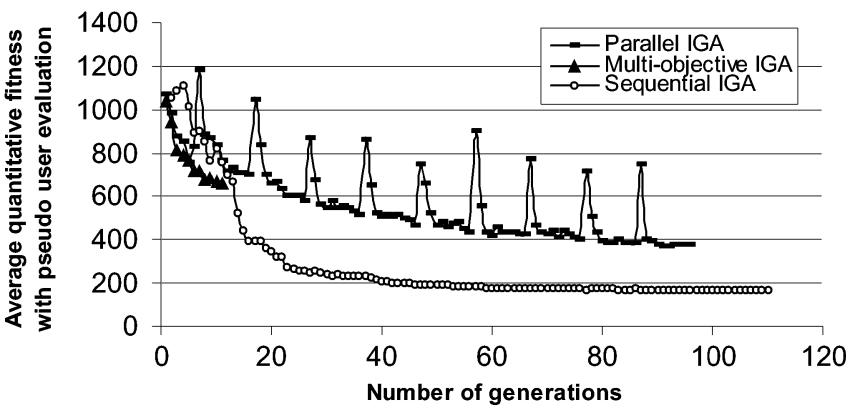

(b)

Fig. 8. Change of average (a) qualitative and (b) quantitative fitness in sequential IGA, multiobjective IGA, and parallel IGA with pseudouser evaluation.

resulting designs were displayed to the users, the users overwhelmingly preferred designs obtained by the algorithms with user given fitness assignment.

\section{Key OBSERVATIONS AND DISCUSSION}

The aim of this work was to provide a complementary design framework that formally brings qualitative and quantitative design criteria together. The algorithms that follow the principles of the framework were tested by the ergonomic chair design problem. A truly ergonomic design process needs live experimentation; providing a completely computer-based ergonomic design optimization framework is neither desirable nor possible. A more complete understanding of the problem can be achieved by formally incorporating qualitative criteria into ergonomic design, and using both qualitative and quantitative criteria to complement the solution search instead of engaging in an epistemological argument over which view is better. The multiobjective philosophy helps the designer to understand the solution landscape. Within our framework, the use of GA provides flexible and simple design encoding and optimization. Interactive designer involvement provides a simple yet efficient way to include qualitative concerns into the design process. As the user modifies individuals and reinserts them into the population, and gains information about the behavior of the multiobjective search space, the framework becomes a two-way informative process.

The main advantage of parallel IGA is its flexibility to accommodate more than one population. The population size in multiobjective IGA needs to be constant as multiple objectives are handled simultaneously in each design. In parallel IGA, limiting the population of qualitatively evaluated designs and increasing the number of quantitatively evolved designs is possible. This makes sure that the time spent by the human during design evaluation is well utilized. As more individuals and generations can be evolved in the quantitatively optimized island, we can get a better fitness in that role. As the populations are on different islands, the parallel IGA does not induce a fight between the two objectives, qualitative and quantitative, as in the case of sequential IGA. In sequential IGA, increasing the size of the population when it is evaluated by a quantitative objective function is also possible.

The multiobjective IGA and the parallel IGA are significantly better than the sequential IGA in terms of the final solutions achieved. Sequential optimization, although it is the most commonly used ergonomic optimization method, did not give satisfactory results in dealing with objectives of different nature together. On the other hand, the multiobjective IGA and the parallel IGA were both seen to be satisfactory for incorporating multiple objectives, and for dealing with qualitative and quantitative design objectives, and seem to be promising approaches.

Some of the challenges observed during experimentation include user fatigue and human inconsistency. User inconsistency can result from: the inability of the user to provide a rating consistent with ratings given for other designs; evaluation of the design with a different perspective each time; or simply changing his/her mind. This makes qualitative convergence very difficult and, hence, the user must be well informed on how the ratings should be given.

User fatigue is the inability of the designer to assess a large number of designs over a large number of generations due to psychological or physical exhaustion. This prevents the evaluation of a large number of generations or large populations by the human user. Various proposals on tackling user fatigue are currently being researched in the IEC community, and have recently been reviewed by one of us [5]. Wang and Takagi proposed a neural network-based mechanism to predict user evaluation, thereby eliminating the need to involve the user in the 
evaluation of every generation [24]. A more recent development is the comparison of online learning within IEC using a fuzzy rule base, radial basis functions, and case-based reasoning by Machwe et al. [25]. They concluded that case-based reasoning is the way forward for online learning in IEC-based optimization.

The current framework can be used with the multiobjective IGA or parallel IGA algorithms to make real-life experimentation, where the chair shape suggested by the computer is actually built and experienced by the user before a rating is provided.

Multiobjective optimization design philosophy can be used to optimize an inclusive design that takes into account varying sizes in a given population of sitters and achieves a compromise design.

The same philosophy can be used to add further qualitative objectives that separately denote the satisfaction of a number of users and the expert judgment of an ergonomics designer.

The framework can be applied to other visual ergonomic design problems that have qualitative and quantitative characteristics.

An existing population of chair designs can be used as a starting point, so that previous designs are used as parent solutions.

As part of our future work, we anticipate the development of a hybrid algorithm in which the strengths of parallel and multiobjective IGA will be combined.

\section{CONCLUSION}

Both qualitative and quantitative views are part of the ergonomic design process. However, the effective formalization and incorporation of both of these influences is a problem that still had to be solved when we began this work.

Here, we have proposed the IMODO framework and developed three algorithmic approaches that followed the principles of the framework. These are the sequential, parallel, and multiobjective IGA. We selected the ergonomic chair design problem for testing the framework. The results obtained from the three algorithms were compared in terms of qualitative and quantitative fitness convergences, diversity of designs, and user preferences among final sets of solutions. The qualitative fitness convergence was best with multiobjective IGA, followed by parallel IGA and sequential IGA. The quantitative fitness convergence was best with parallel IGA, followed by sequential IGA and multiobjective IGA. Users mostly preferred the results obtained from the multiobjective IGA, followed by the parallel IGA, while sequential IGA results were not liked at all. Diversity increased from sequential IGA to parallel IGA to multiobjective IGA.

Our work provides a complementary ergonomic design framework that brings qualitative and quantitative aspects of ergonomic design together. Future work will focus on improving the framework with hybrid algorithms.

\section{REFERENCES}

[1] A. Brintrup, J. Ramsden, and A. Tiwari, "Integrated qualitativeness in design by multi-objective optimization and interactive evolutionary computation," in Proc. IEEE Cong. Evol. Comput. (CEC2005), Edinburgh, U.K., Sep. 2005, vol. 3, pp. 2154-2160.
[2] A. Brintrup, J. Ramsden, and A. Tiwari, "A review on design optimization with interactive evolutionary computation," in Proc. Online World Soft Comput. Conf., Sep. 2005.

[3] B. Shackel, K. D. Chidsey, and P. Shipley, "The assessment of chair comfort," Ergonomics, vol. 12, no. 2, pp. 269-306, 1969.

[4] K. Deb, "Multi-objective optimization using evolutionary algorithms," in Wile Interscience Series in Systems and Optimization, S. Ross and R. Weber, Eds. Chichester, U.K.: Wiley, 2001.

[5] H. Takagi, "Interactive evolutionary computation: Fusion of the capabilities of EC optimization and human evaluation," Proc. IEEE, vol. 89, no. 9, pp. 1275-1296, Sep. 2001.

[6] I. C. Parmee, "Improving problem definition through Interactive evolutionary computation," in Artificial Intelligence For Engineering Design, Analysis and Manufacturing. Cambridge, U.K.: Cambridge Univ. Press, 2002, vol. 16, pp. 185-202, 3.

[7] V. Oduguwa, A. Tiwari, and R. Roy, "Handling integrated quantitative and qualitative search space in a real world optimization problem," in Proc. 2003 Congr. Evol. Comput., Canberra, ACT, Australia, Dec. 2003 , pp. 1222-1229.

[8] T. Inoue, T. Furuhashi, and M. Fujii, "Development of nurse scheduling support system using interactive evolutionary algorithms," in Proc. IEEE Int. Conf. Syst., Man, Cybern., Tokyo, Japan, Oct. 1999, vol. 5 , pp. 533-537.

[9] D. Levine, M. Facello, and P. Hallstrom, "Stalk: An interactive system for virtual molecular docking," IEEE Computational Sci. Eng., vol. 4 no. 2, pp. 55-65, Apr. 1997.

[10] M. R. N. Shackelford and D. W. Corne, "Collaborative evolutionary multi-project resource scheduling," in Proc. Congr. Evol. Comput., Seoul, Korea, May 2001, pp. 1131-1138.

[11] R. Kamalian, H. Takagi, and A. Agogino, "Optimized design of MEMS by evolutionary multi-objective optimization with interactive evolutionary computation," in Proc. Genetic Evol. Comput. Conf., Seattle, WA, Jun. 2004, pp. 1030-1041.

[12] A. Brintrup, A. Tiwari, and J. Gao, "Handling qualitativeness in evolutionary multiple objective engineering design optimization," Enformatica, vol. 1, no. 1, pp. 236-240, 2004.

[13] A. Brintrup, H. Takagi, A. Tiwari, and J. Ramsden, "Evaluation of sequential, multi-objective and parallel interactive genetic algorithms for multi-objective optimization problems," J. Biol. Phys. Chem., vol. 6, no. 3, pp. 137-146, 2006.

[14] A. Machwe, I. C. Parmee, and J. C. Miles, "Integrating aesthetic criteria with a user-centric evolutionary system via a component based design representation," in Proc. Int. Conf. Eng. Design, Melbourne, Australia, 2005.

[15] A. Machwe and I. C. Parmee, "Integrating aesthetic criteria with evolutionary processes in complex, free-form design - an initial investigation," in Proc. IEEE Cong. Evol. Comput. (CEC2006), Jul. 2007, pp. $165-172$.

[16] E. Grandjean, W. Hunting, and M. Pidermann, "VDT workstation design: Preferred settings and their effects," Human Factors, vol. 15, no. 2, pp. 161-175, 1983.

[17] A. R. Tilley, The Measure of Man and Woman: Human Factors in Design, Revised ed. New York: Wiley, 2002.

[18] E. Cantu-Paz, Efficient and Accurate Parallel Genetic Algorithms. Norwell, MA: Kluwer, 2000.

[19] D. A. Van Veldhuizen, J. B. Zydallis, and G. B. Lamont, "Considerations in engineering parallel multiobjective evolutionary algorithms," IEEE Trans. Evol. Comput., vol. 7, no. 2, pp. 144-173, Apr. 2003.

[20] T. Hiroyasu, M. Miki, and S. Watanabe, "The new model of parallel genetic algorithm in multi-objective optimization problems-divided range multi-objective genetic algorithms," in Proc. IEEE Congr. Evol. Comput., La Jolla, CA, Jul. 2000, vol. 1, pp. 333-340.

[21] K. Deb, S. Agrawal, A. Pratap, and T. Meyarivan, "A fast elitist non dominated sorting genetic algorithm for multi objective optimization: NSGA-2," in Proc. Parallel Problem Solving from Nature (PPSN2000), Paris, France, Sep. 2000, pp. 858-862.

[22] H. Takagi, S. Wang, and S. Nakano, "Proposal for a framework for optimizing artificial environments based on physiological feedback," J. Physiol. Anthropology Appl. Human Sci., vol. 24, no. 1, pp. 77-80, Jan. 2005.

[23] A. Machwe, I. C. Parmee, and J. C. Miles, "Multi-objective analysis of a component based representation within an interactive evolutionary design system," in Proc. 7th Int. Conf. Adaptive Comput. Design, Manuf., Bristol, U.K., 2006.

[24] S. Wang and H. Takagi, "Improving the performance of predicting users' subjective evaluation characteristics to reduce their fatigue in IEC," J. Physiological Anthropology Appl Human Sci., vol. 24, no. 1 , pp. 81-85, Jan. 2005. 
[25] A. Machwe and I. C. Parmee, "Introducing Machine Learning within an Interactive Evolutionary Design Environment," in Proc. Design, Dubrovnic, Croatia, May 2006, pp. 1-8.

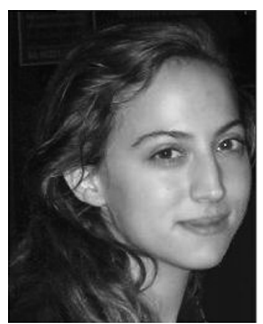

Alexandra Melike Brintrup received the B.Eng. (Hons) degree in manufacturing systems engineering from the University of the West of England, Bristol, U.K., in 2002, the M.Sc. degree in computational techniques for engineering with particular specialization in computer-aided design and the Ph.D. degree in applied sciences from Cranfield University, Bedfordshire, U.K., in 2007. For her Ph.D., she developed an adaptive computing-based framework for bringing qualitative and quantitative design criteria together. During this period she worked for the Vaillant Group and for the ABN AMRO Bank.

She is currently working as a Research Associate at the University of Cambridge, Institute for Manufacturing. She is also involved in the European Sixth Framework PROMISE and BRIDGE projects. Her research focuses on the application of adaptive computing mechanisms to design and manufacturing decision support and optimization. Her research interests include design and manufacturing using interactive evolutionary computing, multiobjective optimization, and the advance of decision support systems in the manufacturing organization.

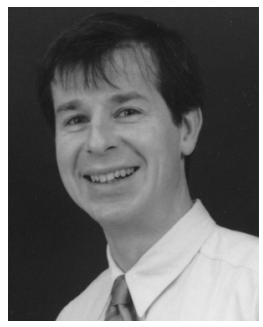

Jeremy Ramsden studied natural sciences at Cambridge University, Cambridge, U.K. and received the Doctorate degree from the Swiss Federal Institute of Technology, Lausanne (EPFL), Switzerland, for work on semiconductor nanoparticles, and was at Princeton University, Princeton, NJ, for postdoctoral studies.

He became a member of the Faculty of Natural Philosophy, Basel University, in 1994. He was appointed to the Chair of Nanotechnology at Cranfield University in 2002, and in 2003 was also appointed Research Director for Bionanotechnology at Cranfield University, Kitakyushu. During the last decade, his main research focus has been the self-assembly of complex biomacromolecules, involving evolving intermolecular and intramolecular interactions; he has explored the use of a similar conceptual approach for modeling various other phenomena in biology and chemistry. He has published about 150 research articles, several books, has chaired several international conferences on biomolecular interactions. He is currently Editor-in-Chief of the Journal of Biological Physics and Chemistry (JBPC) and of Nanotechnology Perceptions: a Review of Ultraprecision Engineering and Nanotechnology. In 1993, he founded, and still leads, the MEMOCS Academic/Industrial Network of Excellence working on integrated-optical membrane-based sensors for medical and environmental applications.

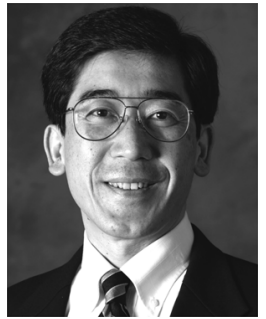

Hideyuki Takagi (M'91) received the B.S. and M.S. degrees from the Kyushu Institute of Design (KID), Fukuoka, Japan, in 1979 and 1981, and the Ph.D. degree in engineering from the Toyohashi University of Technology, Toyohashi, Japan, in 1991.

His professional career includes a Researcher at the Central Research Laboratories, Matsushita Electric Industrial Company, Ltd., from 1981 to 1995, a Visiting Industrial Fellow at UC Berkeley from 1991 to 1993, and an Associate Professor at KID from 1995 to 2003 and at Kyushu University since 2003. He is interested in the cooperative technology of neural networks, fuzzy systems, and evolutionary computation and is one of the most active researchers in interactive evolutionary computation area. He made significant contributions to the start of neurofuzzy system since 1988 .

Dr. Takagi received the Shinohara Memorial Young Engineer Award from the Institute of Electrical, Information, and Communication Engineers (IEICE) in 1989, the Best Paper Awards from KES'97 and IIZUKA'98 Conferences, the PC Best Paper Awards from ICOIN-15 Conference in 2001, and the Paper Award from the Japan Society for Fuzzy Theory and Intelligent Informatics (SOFT) in 2004. He also received the Distinguished Service Award, the Outstanding Contribution Award, and the Best Associate Editor Award from the Slovak Artificial Intelligence Society in 2002, and IEEE SMC Society in 2003 and 2005, respectively. He serves/served as a Vice-President for the IEEE SMC Society from 2006 to 2007, a Board of Governors Member from 2004 to 2006, and an AdCom Member from 2001 to 2003. He also served for SOFT as an Executive Committee member from 1999 to 2001.

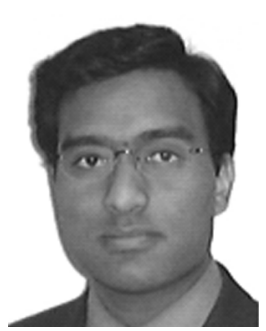

Ashutosh Tiwari (SM'04) is leading the research in applied soft computing within the Decision Engineering Centre, Cranfield University, Bedfordshire, U.K., and has developed new research areas in the application of soft computing to product and process design. He is an Editorial Board Member of the Applied Soft Computing Journal from Elsevier and an Associate Editor of the International Journal of Applied Fuzzy Sets Theory from Research Sciences Press. He has published extensively in his research career: over 58 research papers in refereed journals, conferences and books, and an edited book on applied soft computing. 